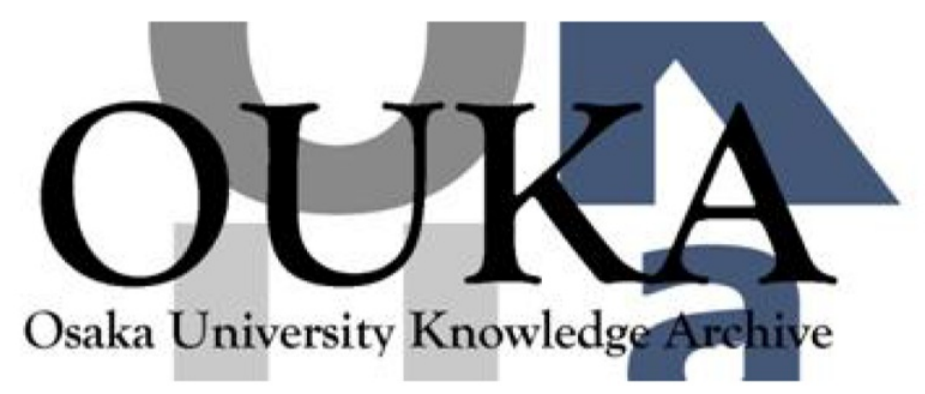

\begin{tabular}{|c|l|}
\hline Title & Reversibility in quantum measurement processes \\
\hline Author(s) & Ueda, Masahito; Kitagawa, Masahiro \\
\hline Citation & Physical Review Letters. 68(23) p. 3424-p. 3427 \\
\hline Issue Date & $1992-06-08$ \\
\hline oaire:version VoR \\
\hline URL & https://hdl. handle.net/11094/77657 \\
\hline rights & $\begin{array}{l}\text { Copyright (1992) by the American Physical } \\
\text { Society }\end{array}$ \\
\hline Note & \\
\hline
\end{tabular}

Osaka University Knowledge Archive : OUKA

https://ir. Library. osaka-u. ac. jp/

Osaka University 


\title{
Reversibility in Quantum Measurement Processes
}

\author{
Masahito Ueda and Masahiro Kitagawa \\ NTT Basic Research Laboratories, Musashino, Tokyo 180, Japan
}

(Received 2 March 1992)

\begin{abstract}
A measurement process is logically reversible if the premeasurement density operator of the measured system can be calculated from the postmeasurement density operator and the readout of the measuring apparatus. We show that the continuous-measurement version of a quantum counter, unlike the conventional photon counter, performs a logically reversible measurement. The physical origin for such a distinction is found to be sensitivity to vacuum field fluctuations.

PACS numbers: $42.50 . \mathrm{Dv}, 03.65 . \mathrm{Bz}$
\end{abstract}

The irreversibility of a quantum measurement has rarely if ever been questioned, in part because a measurement process involves state reduction and this reduction usually singles out one of the possible eigenvalues of an observable, eliminating all other possibilities. This kind of measurement (i.e., a sharp measurement), however, requires a strong coupling between the measuring apparatus and the measured system. If the coupling is not strong enough, a single measurement can determine only an envelope that peaks unsharply around some value. Since such an unsharp measurement does not exclude possibilities other than this value, we may wonder if there is a quantum measurement that lets us reproduce the initial density operator from the postmeasurement density operator and the readout of the measuring apparatus. This Letter answers this question affirmatively in the context of the photon-number measurement.

A continuous photodetection process can be decomposed into a time sequence of two fundamental processes: no-count and one-count processes [1]. A no-count process is one in which no photons are detected, and the state evolution during a no-count process is given (in the interaction representation) by [2]

$$
\hat{\rho}(t+\tau)=\frac{\exp \left(-\frac{\lambda}{2} \hat{a}^{\dagger} \hat{a} \tau\right) \hat{\rho}(t) \exp \left(-\frac{\lambda}{2} \hat{a}^{\dagger} \hat{a} \tau\right)}{\operatorname{Tr}\left[\hat{\rho}(t) \exp \left(-\lambda \hat{a}^{\dagger} \hat{a} \tau\right)\right]},
$$

where $\hat{\rho}(t)$ is the initial density operator of the photon field, $\hat{a}^{\dagger}$ and $\hat{a}$ are the photon creation and annihilation operators, and $\lambda$ is the coupling constant between the photodetector and the photon field. The state evolution represented by Eq. (1) is nonunitary because it is accompanied by information readout "no count" and projection of the state must therefore be made throughout the process. Nevertheless Eq. (1) can be inverted to give

$$
\hat{\rho}(t)=\frac{\exp \left(\frac{\lambda}{2} \hat{a}^{\dagger} \hat{a} \tau\right) \hat{\rho}(t+\tau) \exp \left(\frac{\lambda}{2} \hat{a}^{\dagger} \hat{a} \tau\right)}{\operatorname{Tr}\left[\hat{\rho}(t+\tau) \exp \left(\lambda \hat{a}^{\dagger} \hat{a} \tau\right)\right]} .
$$

Equation (2) gives the initial density operator $\hat{\rho}(t)$ in terms of the final density operator $\hat{\rho}(t+\tau)$ and the knowledge that no photons were detected between $t$ and $t+\tau$. The no-count process is therefore logically reversible.
A one-count process is one in which one photon is detected during an infinitesimal time $d t$. The photon density operator immediately after the one-count process is given by $[1,2]$

$$
\hat{\rho}\left(t^{+}\right)=\frac{\hat{a} \hat{\rho}(t) \hat{a}^{\dagger}}{\operatorname{Tr}\left[\hat{\rho}(t) \hat{a}^{\dagger} \hat{a}\right]},
$$

where $t^{+}$denotes a time infinitesimally later than $t$. This equation cannot be inverted: that is, the premeasurement state $\hat{\rho}(t)$ cannot be reproduced from the postmeasurement state $\hat{\rho}\left(t^{+}\right)$. This is because a conventional photodetector does not respond to the vacuum state, so $\hat{\rho}\left(t^{+}\right)$ does not contain information about the vacuum state of the premeasurement density operator-that is, the information given in the number-state basis by $\rho_{0 n}(t)$ and $\rho_{n 0}(t)(n=0,1,2, \ldots)$.

Thus we find that the conventional photon counter does not perform logically reversible measurement, and that the physical origin of this irreversibility lies in the counter's insensitivity to the vacuum state. Sensitivity to the vacuum state is therefore essential for reversible measurement.

The concept of logical reversibility proposed here is fundamentally important because it gives a criterion for deciding whether or not the system's information is conserved during a measurement process: If the measurement process is logically reversible, the amount of information contained in the postmeasurement density operator plus the readout of the measuring apparatus is exactly equal to the amount of information contained in the initial density operator. In most measurement schemes, some information is lost and the initial density operator therefore cannot be calculated from the postmeasurement density operator and the readout of the measuring apparatus. One might conclude from this that the nonunitary state reduction associated with information readout entails loss of information, thereby making a quantum measurement irreversible. We show here, however, that this is not so if we understand reversibility from the viewpoint of conservation of information.

The model we propose for reversible photon counting is a continuous-measurement version of a quantum 
counter that is sensitive to the vacuum state. In a typical quantum counter (Fig. 1) [3], an atom is brought into a metastable state $c$ by optical pumping $(a \rightarrow b)$ followed by nonradiative decay $(b \rightarrow c)$. This device is sensitive to a photon field whose energy matches the energy difference $\hbar \omega_{0}$ between levels $c$ and $d$. Because the atom is put into an excited state $c$, it will respond not only to photons but also to vacuum field fluctuations by spontaneous radiative decay. Level $d$ is assumed to be very short-lived, so that as soon as the atom transits from $c$ to $d$, it decays into the ground state $a$, producing a photon of energy $\hbar \omega$ that differs from $\hbar \omega_{0}$. Detecting a photon with energy $\hbar \omega$ thus implies either stimulated or spontaneous emission between levels $c$ and $d$. The quantum counter was first devised for detecting infrared photons [4], and it was later pointed out that it could also be used to measure antinormal-order correlation functions [3]. In the present Letter we use this device to provide a continuous measurement of photon number, and we show that it works as a logically reversible quantum counter.

The reversible photon counter is the one in which excited two-level atoms are injected one by one into an optical cavity and the level of each atom coming out of the cavity is measured. An output atom in the excited state means that the detector responded neither to photons nor to the vacuum field (no-count process), and an output atom in the ground state means that the atom responded either to a photon or to the vacuum field (onecount process). In this model, the measurement of the atomic level replaces the measurement of a second photon of energy $\hbar \omega$ in quantum counters, but an essential feature of quantum counters - sensitivity to vacuum field fluctuations-is retained. Moreover, since at most one photon is detected at one time, this device performs unsharp measurement. We will show that the combination

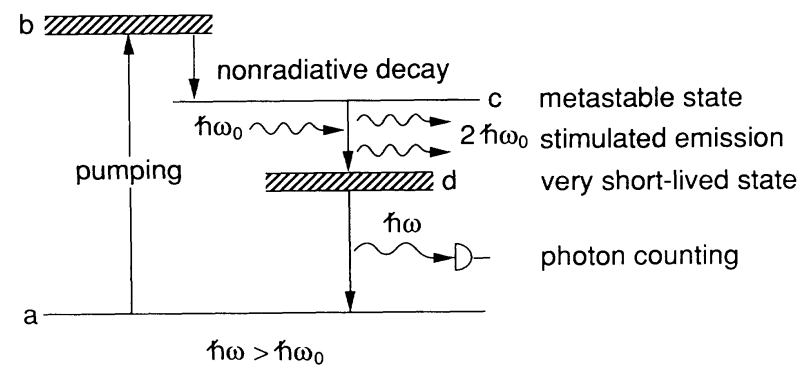

FIG. 1. Schematic illustration of a quantum counter. An atom is prepared in a metastable state $c$ by optical pumping $(a \rightarrow b)$ followed by nonradiative decay $(b \rightarrow c)$. The radiative decay $(c \rightarrow d)$ stimulated by either photons or vacuum field fluctuations is immediately followed by another radiative decay $(d \rightarrow a)$ whose occurrence is detected by a conventional photon counter.

of these two features makes the measurement process logically reversible.

The interaction Hamiltonian between the photon field and the atom is given by $\hat{H}_{\text {int }}=\hbar g\left(\hat{a} \hat{\sigma}_{+}+\hat{\sigma}_{-} \hat{a}^{\dagger}\right)$, where $\hat{\sigma}_{+}$and $\hat{\sigma}_{-}$are the level-raising and level-lowering operators and $g$ is a coupling constant. For the ground state $|g\rangle_{a}$ and the excited state $|e\rangle_{a}$ of an atom, they operate as $\hat{\sigma}_{+}|g\rangle_{a}=|e\rangle_{a}, \hat{\sigma}_{+}|e\rangle_{a}=0, \hat{\sigma}_{-}|g\rangle_{a}=0$, and $\hat{\sigma}_{-}|e\rangle_{a}$ $=|g\rangle_{a}$. Since the incident atom is excited, the initial state of the combined atom-field system is given by

$$
\hat{\rho}_{a-f}\left(t_{0}\right)=|e\rangle_{a a}\langle e| \otimes \hat{\rho}_{f}\left(t_{0}\right),
$$

where $\hat{\rho}_{f}\left(t_{0}\right)$ is the initial density operator of the photon field. We assume that each atom interacts with the photon field for a very short time $\Delta t$. Thus, the time evolution of the combined atom-field system can be calculated perturbatively:

$$
\hat{\rho}_{a-f}\left(t_{0}+\Delta t\right)=\hat{\rho}_{a-f}\left(t_{0}\right)+\sum_{m=1}^{\infty} \int_{t_{0}}^{t_{0}+\Delta t} d t_{1} \int_{t_{0}}^{t_{1}} d t_{2} \ldots \int_{t_{0}}^{t_{m-1}} d t_{m}\left[\hat{H}_{\mathrm{int}}\left(t_{1}\right),\left[\hat{H}_{\mathrm{int}}\left(t_{2}\right), \ldots,\left[\hat{H}_{\mathrm{int}}\left(t_{m}\right), \hat{\rho}_{a-f}\left(t_{0}\right)\right] \cdots\right]\right]
$$

where $[\hat{A}, \hat{B}]=\hat{A} \hat{B}-\hat{B} \hat{A}$. Substituting Eq. (4) into Eq. (5) and keeping terms up to second order in the coupling constant, we obtain

$$
\begin{aligned}
\hat{\rho}_{a-f}\left(t_{0}+\Delta t\right)= & i g \Delta t\left[\hat{\rho}_{f}\left(t_{0}\right) \hat{a} \otimes|e\rangle_{a a}\left\langle g\left|-\hat{a}^{\dagger} \hat{\rho}_{f} \otimes\right| g\right\rangle_{a a}\langle e|\right] \\
& +\left[\hat{\rho}_{f}\left(t_{0}\right)-\frac{(g \Delta t)^{2}}{2}\left[\hat{a} \hat{a}^{\dagger} \hat{\rho}_{f}\left(t_{0}\right)+\hat{\rho}_{f}\left(t_{0}\right) \hat{a} \hat{a}^{\dagger}\right]\right] \otimes|e\rangle_{a a}\left\langle e\left|+(g \Delta t)^{2} \hat{a}^{\dagger} \hat{\rho}_{f}\left(t_{0}\right) \hat{a} \otimes\right| g\right\rangle_{a a}\langle g| .
\end{aligned}
$$

The time evolution of the photon field depends on the result of the atomic-state measurement. If the atom is found in the ground state (i.e., the one-count process), the photon state immediately after measurement, $\hat{\rho}_{f}\left(t_{0}\right.$ $+\Delta t$ ), is given by

$$
\hat{\rho}_{f}\left(t_{0}+\Delta t\right)=\frac{\operatorname{Tr}_{a}\left[\hat{\rho}_{a-f}\left(t_{0}+\Delta t\right) \hat{\rho}_{g}^{\text {(read })}\right]}{\operatorname{Tr}_{a-f}\left[\hat{\rho}_{a-f}\left(t_{0}+\Delta t\right) \hat{\rho}_{g}^{\text {(read })}\right]}
$$

where $\operatorname{Tr}_{a}$ and $\operatorname{Tr}_{a-f}$ denote trace operations over the atomic system and over the atom-field system, and $\hat{\rho}_{g}^{(\text {read })}=\hat{1}_{f} \otimes|g\rangle_{a a}\langle g|$ is the probability-operator measure [5] for the coupled system. Here $\hat{1}_{f}$ denotes the identity operator for the photon field. Substituting Eq. (6) and $\hat{\rho}_{g}^{(\text {read) }}$ into Eq. (7) yields for the one-count process

$$
\hat{\rho}_{f}\left(t_{0}+\Delta t\right)=\frac{\hat{a}^{\dagger} \hat{\rho}_{f}\left(t_{0}\right) \hat{a}}{\operatorname{Tr}_{f}\left[\hat{\rho}_{f}\left(t_{0}\right) \hat{a} \hat{a}^{\dagger}\right]}
$$


where $\operatorname{Tr}_{f}$ denotes a trace operation over the photon field. If the output atom is in the excited state (i.e., the nocount process), the probability-operator measure is given by $\hat{\rho}_{e}^{(\mathrm{read})}=\hat{1}_{f} \otimes|e\rangle_{a a}\langle e|$. The photon density operator immediately after the no-count process is therefore given by

$\hat{\rho}_{f}\left(t_{0}+\Delta t\right)=\frac{\exp \left(-\frac{\lambda}{2} \hat{a} \hat{a}^{\dagger} \Delta t\right) \hat{\rho}_{f}\left(t_{0}\right) \exp \left(-\frac{\lambda}{2} \hat{a} \hat{a}^{\dagger} \Delta t\right)}{\operatorname{Tr}_{f}\left[\hat{\rho}_{f}\left(t_{0}\right) \exp \left(-\lambda \hat{a} \hat{a}^{\dagger} \Delta t\right)\right]}$,

where $\lambda \equiv g^{2} \Delta t$. Equations (8) and (9) show how the photon state evolves according to the result of measurement. Equation (8) describing the one-count process can be inverted to give

$$
\hat{\rho}_{f}\left(t_{0}\right)=\frac{\frac{1}{\hat{n}+1} \hat{a} \hat{\rho}_{f}\left(t_{0}+\Delta t\right) \hat{a}^{\dagger} \frac{1}{\hat{n}+1}}{\operatorname{Tr}_{f}\left[\hat{\rho}_{f}\left(t_{0}+\Delta t\right) \hat{a}^{\dagger}\left(\frac{1}{\hat{n}+1}\right)^{2} \hat{a}\right]},
$$

where $\hat{n} \equiv \hat{a}^{\dagger} \hat{a}$. This equation shows that the premeasurement photon density operator $\hat{\rho}_{f}\left(t_{0}\right)$ can be calculated if we find the postmeasurement photon density operator $\hat{\rho}_{f}\left(t_{0}+\Delta t\right)$ and if we know that the output atom was in the ground state. The one-count process is therefore logically reversible. And the no-count process is logically reversible because Eq. (9) can also be inverted:

$$
\hat{\rho}_{f}\left(t_{0}\right)=\frac{\exp \left(\frac{\lambda}{2} \hat{a} \hat{a}^{\dagger} \Delta t\right) \hat{\rho}_{f}\left(t_{0}+\Delta t\right) \exp \left(\frac{\lambda}{2} \hat{a} \hat{a}^{\dagger} \Delta t\right)}{\operatorname{Tr}_{f}\left[\hat{\rho}_{f}\left(t_{0}+\Delta t\right) \exp \left(\lambda \hat{a} \hat{a}^{\dagger} \Delta t\right)\right]} .
$$

Since continuous measurement consists of a sequence of one-count and no-count processes $[2,6]$ and both of these elementary processes are logically reversible, the whole measurement process should also be logically reversible. Here we explicitly prove this. For convenience of description, let us define the following two superoperators:

$\mathcal{J} \hat{\rho}_{f}(t) \equiv \lambda \hat{a}^{\dagger} \hat{\rho}_{f}(t) \hat{a}$

$\mathcal{S}_{\tau} \hat{\rho}_{f}(t) \equiv \exp \left(-\frac{\lambda}{2} \hat{a} \hat{a}^{\dagger} \tau\right) \hat{\rho}_{f}(t) \exp \left(-\frac{\lambda}{2} \hat{a} \hat{a}^{\dagger} \tau\right)$

Suppose that the measurement process starts at $t_{0}$ and ends at $t_{0}+\tau$, and that $m$ photons are detected at times $t_{1}, t_{2}, \ldots, t_{m}\left(t_{0} \leq t_{1}<t_{2}<\cdots<t_{m} \leq t_{0}+\tau\right)$. Let $\hat{\rho}_{f}\left(t_{1}, t_{2}, \ldots, t_{m} ; t_{0}, t_{0}+\tau\right)$ be the photon density operator immediately after the measurement process. It can be expressed in terms of superoperators $\mathcal{J}$ and $\mathcal{S}_{\tau}$ as [2]

$$
\hat{\rho}_{f}\left(t_{1}, t_{2}, \ldots, t_{m} ; t_{0}, t_{0}+\tau\right)=\frac{\mathcal{S}_{t_{0}+\tau-t_{m}} \mathcal{J} \mathcal{S}_{t_{m}-t_{m-1}} \mathcal{J} \cdots \mathcal{S}_{t_{2}-t_{1}} \mathcal{J} \mathcal{S}_{t_{1}-t_{0}} \hat{\rho}_{f}\left(t_{0}\right)}{\operatorname{Tr}_{f}\left[\mathcal{S}_{t_{0}+\tau-t_{m}} \mathcal{J} \mathcal{S}_{t_{m}-t_{m-1}} \mathcal{J} \cdots \mathcal{S}_{t_{2}-t_{1}} \mathcal{J} \mathcal{S}_{t_{1}-t_{0}} \hat{\rho}_{f}\left(t_{0}\right)\right]}
$$

From Eqs. (12) and (13), we obtain

$$
\begin{aligned}
\mathcal{S}_{t_{0}+\tau-t_{m}} \mathcal{J} \mathcal{S}_{t_{m}-t_{m-1}} \mathcal{J} \cdots \mathcal{S}_{t_{2}-t_{1}} \mathcal{J} \mathcal{S}_{t_{1}-t_{0}} \hat{\rho}_{f}\left(t_{0}\right) & \\
= & =\lambda^{m} \exp \left(\lambda \sum_{i=1}^{m} t_{i}\right) \exp \left(-\frac{\lambda}{2} \hat{a} \hat{a}^{\dagger} \tau\right)\left(\hat{a}^{\dagger}\right)^{m} \hat{\rho}_{f}\left(t_{0}\right) \hat{a}^{m} \exp \left(-\frac{\lambda}{2} \hat{a} \hat{a}^{\dagger} \tau\right) .
\end{aligned}
$$

The trace of this quantity gives the probability distribution $\mathcal{P}\left(t_{1}, t_{2}, \ldots, t_{m} ; t_{0}, t_{0}+\tau\right)$ that one-count processes occur only at times $t_{1}, t_{2}, \ldots, t_{m}$ :

$$
\mathcal{P}\left(t_{1}, t_{2}, \ldots, t_{m} ; t_{0}, t_{0}+\tau\right)=\lambda^{m} \exp \left(\lambda \sum_{i=1}^{m} t_{i}\right) \operatorname{Tr}_{f}\left[\hat{\rho}_{f}\left(t_{0}\right) \hat{a}^{m} \exp \left(-\lambda \hat{a} \hat{a}^{\dagger} \tau\right)\left(\hat{a}^{\dagger}\right)^{m}\right] .
$$

The time dependence $\exp \left(\lambda \sum_{i=1}^{m} t_{i}\right)$, which appears in the probability distribution (16), cancels out in forming the ratio on the right-hand side of Eq. (14), and the density operator after the measurement process no longer depends on the times when photocounts are registered, $t_{i}\left(i=1,2, \ldots, t_{m}\right)$. Thus we obtain

$$
\hat{\rho}_{f}\left(m ; t_{0}, t_{0}+\tau\right)=\frac{\exp \left(-\frac{\lambda}{2} \hat{a} \hat{a}^{\dagger} \tau\right)\left(\hat{a}^{\dagger}\right)^{m} \hat{\rho}_{f}\left(t_{0}\right) \hat{a}^{m} \exp \left(-\frac{\lambda}{2} \hat{a} \hat{a}^{\dagger} \tau\right)}{\operatorname{Tr}_{f}\left[\hat{\rho}_{f}\left(t_{0}\right) \hat{a}^{m} \exp \left(-\lambda \hat{a} \hat{a}^{\dagger} \tau\right)\left(\hat{a}^{\dagger}\right)^{m}\right]},
$$

where $\hat{\rho}_{f}\left(m ; t_{0}, t_{0}+\tau\right) \equiv \hat{\rho}_{f}\left(t_{1}, t_{2}, \ldots, t_{m} ; t_{0}, t_{0}+\tau\right)$. Equation (17) describes the time evolution of the photon density operator under the influence of the continuous measurement performed by a quantum counter. The evolution is nonunitary because in continuous measurement both the atom-field interaction and the projection associated with measurement proceed simultaneously. The crucial point here is that nonunitary state evolution does not mean that the measurement process is logically irreversible. Unlike continuous measurement by a conventional photon counter $[2,7]$, Eq. (17) can be inverted. By inspection we obtain

$$
\hat{\rho}_{f}\left(t_{0}\right)=\frac{\left(\frac{1}{\hat{n}+1} \hat{a}\right)^{m} \exp \left(\frac{\lambda}{2} \hat{a} \hat{a}^{\dagger} \tau\right) \hat{\rho}_{f}\left(m ; t_{0}, t_{0}+\tau\right) \exp \left(\frac{\lambda}{2} \hat{a} \hat{a}^{\dagger} \tau\right)\left(\hat{a}^{\dagger} \frac{1}{\hat{n}+1}\right)^{m}}{\operatorname{Tr}_{f}\left[\hat{\rho}_{f}\left(m ; t_{0}, t_{0}+\tau\right) \exp \left(\frac{\lambda}{2} \hat{a} \hat{a}^{\dagger} \tau\right)\left(\hat{a}^{\dagger} \frac{1}{\hat{n}+1}\right)^{m}\left(\frac{1}{\hat{n}+1} \hat{a}\right)^{m} \exp \left(\frac{\lambda}{2} \hat{a} \hat{a}^{\dagger} \tau\right)\right]} .
$$


Because the premeasurement photon density operator can be calculated from the postmeasurement photon density operator and the result of measurement (i.e., how many photocounts are registered), we find that the proposed continuous-measurement version of a quantum counter performs logically reversible measurement.

What happens to the state evolution if the quantum counter is switched on but the output atomic state is not measured? (We shall refer to this process as a nonreferring measurement process [2].) We will show here that the state evolution drastically changes and that logical reversibility is no longer retained.

In the nonreferring measurement process we do not refer to either the number of photocounts or the times of their occurrences. The superoperator $\mathcal{T}_{\tau}$ describing this process is therefore given by [2]

$$
\mathcal{T}_{\tau}=\sum_{m=0}^{\infty} \int_{t_{0}}^{t_{0}+\tau} d t_{m} \int_{t_{0}}^{t_{m}} d t_{m-1} \cdots \int_{t_{0}}^{t_{2}} d t_{1} \mathcal{S}_{t_{0}+\tau-t_{m}} \mathcal{J} \mathcal{S}_{t_{m}-t_{m-1}} \mathcal{J} \cdots \mathcal{S}_{t_{2}-t_{1}} \mathcal{J} \mathcal{S}_{t_{1}-t_{0}}
$$

Substituting Eq. (15) into this equation yields

$$
\mathcal{T}_{\tau} \hat{\rho}_{f}\left(t_{0}\right)=\sum_{m=0}^{\infty} \frac{e^{\lambda \tau}-1}{m !} \exp \left(-\frac{\lambda}{2} \hat{a} \hat{a}^{\dagger} \tau\right)\left(\hat{a}^{\dagger}\right)^{m} \hat{\rho}_{f}\left(t_{0}\right) \hat{a}^{m} \exp \left(-\frac{\lambda}{2} \hat{a} \hat{a}^{\dagger} \tau\right)
$$

Since the trace of this quantity is unity, the time evolution of the photon density operator is given by

$$
\hat{\rho}_{f}\left(t_{0}+\tau\right)=\sum_{m=0}^{\infty} \frac{\left(e^{\lambda \tau}-1\right)^{m}}{m !} \exp \left(-\frac{\lambda}{2} \hat{a} \hat{a}^{\dagger} \tau\right)\left(\hat{a}^{\dagger}\right)^{m} \hat{\rho}_{f}\left(t_{0}\right) \hat{a}^{m} \exp \left(-\frac{\lambda}{2} \hat{a} \hat{a}^{\dagger} \tau\right) .
$$

We note that Eq. (21) is a solution to the master equation for a linear amplifier [8]:

$\frac{d}{d t} \hat{\rho}_{f}(t)=\lambda \hat{a}^{\dagger} \hat{\rho}_{f}(t) \hat{a}-\frac{\lambda}{2}\left[\hat{a} \hat{a}^{\dagger} \hat{\rho}_{f}(t)+\hat{\rho}_{f}(t) \hat{a} \hat{a}^{\dagger}\right]$

The state evolution described by Eq. (22) is obviously irreversible. The physical origin of the irreversibility is the quantum noise added in the process of amplification. In fact, Eq. (21) can be expressed in the Heisenberg representation as [9] $\hat{a}\left(t_{0}+\tau\right)=\sqrt{G} \hat{a}\left(t_{0}\right)+\sqrt{G-1} \hat{d}^{\dagger}$, where $G=e^{\lambda \tau}$ is the power gain and $\hat{d}^{\dagger}$ is a noise operator that satisfies $\langle\hat{d}\rangle=\left\langle\hat{d}^{\dagger}\right\rangle=\left\langle\hat{d}^{\dagger} \hat{d}\right\rangle=0$ and $\left\langle\hat{d} \hat{d}^{\dagger}\right\rangle=1$. The irreversibility in the amplification process is clearly caused by the noise term $\hat{d}^{\dagger}$, which is added unpredictably to the relevant mode and thus degrades the signal-to-noise ratio by $3 \mathrm{~dB}$. In our model, the uncertainty corresponding to the noise term results from our discarding the information about whether the readout is the one-count process or the no-count process.

In general, a quantum measurement process plays two distinct roles with respect to the past and future of the measurement process [10]. With respect to the past, it verifies the predicted probability distribution by a number of measurements for an ensemble. With respect to the future, it produces a new state by a single measurement. Such an asymmetry in the direction of time distinguishes quantum mechanics from classical mechanics. This fact, however, does not imply that a quantum measurement process is not logically reversible. We have presented a concrete model that performs a logically reversible measurement.
A measurement process transfers information from the system of concern to a measuring apparatus. Nonunitary state reduction is associated with information readout, but this Letter shows that a measurement process does not necessarily imply loss of information. This discovery merits further theoretical study.

We thank N. Imoto for valuable discussions at an early stage of this work.

[1] M. D. Srinivas and E. B. Davies, Opt. Acta 28, 981 (1981).

[2] M. Ueda, Quantum Opt. 1, 131 (1989); Phys. Rev. A 41, 3875 (1990).

[3] L. Mandel, Phys. Rev. 152, 438 (1966).

[4] N. Bloembergen, Phys. Rev. Lett. 2, 84 (1959).

[5] C. W. Helstrom, Quantum Detection and Estimation Theory (Academic, New York, 1976).

[6] N. Imoto, M. Ueda, and T. Ogawa, Phys. Rev. A 41, 4127 (1990).

[7] M. Ueda, N. Imoto, and T. Ogawa, Phys. Rev. A 41, 3891 (1990).

[8] It is interesting to note that the state evolution for conventional photon counting in the nonreferring measurement process coincides with a solution to the master equation for a linear absorber (see Ref. [7]).

[9] H. A. Haus and J. A. Mullen, Phys. Rev. 128, 2407 (1962).

[10] L. D. Landau and E. M. Lifshitz, Quantum Mechanics (Pergamon, New York, 1958). 